\title{
5. Die kurzfristige Regulierung von Versorgungsengpässen und Überhängen
}

\section{Entlassungen in der Scbwerindustrie und Arbeitskräftebedarf in der Landwirtschaft}

Bei der Arbeitskräfteplanung und -lenkung kristallisierten sich schon frühzeitig systemimmanente Probleme heraus, die ab Mitte der fünfziger Jahre immer mehr zum Tragen kamen und zu einem prägenden Kennzeichen sozialistischer Planwirtschaft werden sollten. Die Arbeitsverwaltung mußte erkennen, daß ihre Bedarfsplanung unzureichend gewesen war, da sich im sekundären Sektor, vor allem bei der Schwerindustrie, ein Arbeitskräfteüberhang bemerkbar machte. Der vorrangige Ausbau der Grundstoff- und Schwerindustrie zeigte somit deutliche negative Folgewirkungen. Die Zentrale Kommission für Staatliche Kontrolle (ZKSK) meldete zunächst Mitte Januar 1955, daß in einigen Betrieben im Bezirk Dresden "Entlassungen von Arbeitskräften in größerem Umfang vorgenommen werden" 1 . Die Entlassungen gingen in der Regel auf die von den zuständigen Ministerien erstellten Arbeitskräftepläne zurück, die eine Laufzeit von zwölf Monaten hatten: So mußte beispielsweise das VEB Transformatoren- und Röntgenwerk Dresden auf Veranlassung des Ministeriums für Maschinenbau 433 Arbeiter seiner insgesamt 4221 Belegschaftsmitglieder entlassen². Der Bericht listete noch weitere Betriebe auf, bei denen sogenannte Freisetzungen notwendig geworden waren. Von der Entlassungswelle wurden in zunehmenden Maße auch Betriebe der Konsum- und Leichtindustrie erfaßt. Es deutet einiges darauf hin, daß diese Entwicklung auch auf das Bestreben der Staatlichen Plankommission und der Industrieministerien zurückzuführen war, in den Betrieben Arbeitskräftehortung zu verhindern bzw. Arbeiter, die dort nicht mehr für die Produktion benötigt wurden, für andere Aufgaben „umzusetzen“. Den entlassenen Arbeitern konnte jedoch oftmals nicht sofort ein neuer Arbeitsplatz angeboten werden, so daß zumindest vorübergehend mit einem Ansteigen der Erwerbslosenzahlen zu rechnen war³. Bei der Vorbereitung des Volkswirtschaftsplanes ging die SED-Führung für das zweite Quartal 1955 von insgesamt 67800 Entlassungen aus, allein 60000 beim Maschinenbau ${ }^{4}$.

Gleichzeitig wurde ein Arbeitskräftebedarf im primären Sektor gemeldet. Der Mangel an Landarbeitern war für die DDR nichts Neues und hatte auch schon zuvor die Führungsgremien der SED sowie des Ministerrates eingehend beschäftigt. So hatte etwa der Ministerrat auf seiner Sitzung am 2. Mai 1952 ein Maßnahmenbündel verabschiedet, um Arbeitskräfte für die Landwirtschaft bereitzustellen ${ }^{5}$. Allerdings strebte man seinerzeit nicht so sehr die Umsetzung von Arbeitskräften zwischen den verschiedenen volkswirtschaftlichen Sektoren an, sondern die Er-

' BAB, DC 1/1055, Bericht der ZKSK vom 17.1. 1955, S. 1. Die folgenden S. 516-525 habe ich in einer ersten verkürzten Fassung bereits an anderer Stelle veröffentlicht. Vgl. Hoffmann, Im Laboratorium der Planwirtschaft, S. 654-662.

2 BAB, DC 1/1055, Bericht der ZKSK vom 17. 1. 1955, S. 2.

3 BAB, DQ 2/853, Bericht der Abt. Arbeitskraftlenkung vom 22. 1. 1955.

SAPMO, DY 30/J IV 2/2/418, Bl. 30.

BAB, DC $20 \mathrm{I} / 3-105$, Bl. 4. 
weiterung des Beschäftigtenstandes. Es sollten „in höherem Maße arbeitseinsatzfähige Sozialfürsorgeunterstützungsempfänger " in der Landwirtschaft eingesetzt werden, die neben dem gesetzlichen Tariflohn ihre Leistungen aus der Sozialfürsorge weiterhin ungekürzt erhalten sollten ${ }^{6}$. Nach Angaben des Ministeriums für Arbeit waren im Monat Mai 1952 insgesamt 12000 Sozialfürsorgeempfänger entsprechend tätig7, wobei ein großer Teil dieser Gruppe aber schon vor Beginn der Werbeaktion in der Landwirtschaft beschäftigt gewesen sein dürfte. Das Ministerium für Land- und Forstwirtschaft veröffentlichte am 21. März 1955 eine Richtlinie "für den Wettbewerb zur Steigerung der Produktion und Erfüllung des Volkswirtschaftsplanes 1955 in der Landwirtschaft" ${ }^{\text {"8. Eng verbunden mit dieser }}$ Mobilisierungskampagne war die zeitgleich erschienene Richtlinie für die Aktion „Industriearbeiter aufs Land“, die zuvor vom ZK der SED abgesegnet worden war'. Die Durchführung oblag den Räten der Kreise und Bezirke. Diese Aktion besaß nicht nur den skizzierten arbeitsmarktpolitischen Hintergrund (Arbeitskräftemangel), sondern muß auch in enger Verbindung mit der SED-Landwirtschaftspolitik gesehen werden: Der Aufbau der Landwirtschaftlichen Produktionsgenossenschaften (LPG), der zunächst 1953 gestoppt worden war, sollte auf diese Weise wieder etwas vorangetrieben werden ${ }^{10}$. Die Vorstellung, die personelle Überbesetzung in der Industrie zur Stärkung der Landwirtschaft zu nutzen, schien zunächst der richtige Lösungsansatz zu sein, stieß allerdings bei der praktischen Umsetzung auf gewaltige Probleme. Auch hier wurde eine Koordinierungsbereitschaft und -fähigkeit der einzelnen Verwaltungen auf zentraler, bezirklicher und lokaler Ebene vorausgesetzt, die in der zeitlichen und inhaltlichen Abfolge nicht erbracht werden konnte. So war es letztlich auch nicht verwunderlich, daß der Bericht der ZKSK über den Verlauf dieser Aktion aus Sicht der SEDFührung niederschmetternd ausfiel: Im einzelnen wurde kritisiert, daß der Arbeitskräftebedarf in den Kreisen oftmals nicht ermittelt wurde, so daß die Arbeitsverwaltung auch keine verläßlichen Daten für ihre weitere Planung besaß11.

Für die Durchführung der Aktion „Industriearbeiter aufs Land“ wurden nicht nur die staatlichen Verwaltungen auf der zentralen und lokalen Ebene, sondern auch die Massenorganisationen eingebunden. So forderte der Minister für Arbeit und Berufsausbildung Fritz Macher den Vorsitzenden des FDGB-Bundesvorstandes Herbert Warnke am 6. August 1955 auf, unterstützende Maßnahmen einzuleiten $^{12}$. Besonders „ungenügend“ sei die bisherige Werbetätigkeit der Betriebsgewerkschaftsleitungen in den Industriebetrieben gewesen. Noch deutlicher wurde die Staatliche Plankommission (HA Planung der Arbeitskräfte), die bei einem Instrukteureinsatz im Bezirk Halle SED und FDJ in die Kritik mit einschloß.

6 Ebenda, Bl. 10f., Anlage 1 zum Protokoll.

7 Ebenda, Bl. 50-56, Minister für Arbeit, Roman Chwalek, am 9.7. 1952 an den Chef der Regierungskanzlei und Staatssekretär der Regierung, Fritz Geyer.

8 BAB, DQ 2/2124.

- Ebenda.

10 BAB, DO 1/8/175, Bl. 31 f., Schreiben des Ministers für Land- und Forstwirtschaft, Hans Reichelt, am 7. 7. 1955 an den Minister des Innern, Karl Maron.

1 Ebenda, Bl. 40-54, Bericht der ZKSK, Arbeitsgruppe Arbeit und Gesundheitswesen, vom 27.7. 1955.

12 SAPMO, DY $34,45 / 143 / 6058$. 
Sie kam in einem bilanzierenden Bericht zum Ergebnis, daß die Tätigkeit der eingesetzten Kommissionen in den Bezirken und Kreisen "dadurch gehemmt [werde], daß die Vertreter der Partei, des FDGB und der FDJ nicht regelmäßig und nicht aktiv an der Arbeit teilnehmen" würden ${ }^{13}$. Dagegen wies der FDGBBundesvorstand die Verantwortung für den schleppenden Fortgang der Aktion den Industrieministerien $\mathrm{zu}^{14}$.

Obwohl zwischen dem Ministerium für Land- und Forstwirtschaft und einzelnen Industrieministerien Vereinbarungen getroffen worden waren ${ }^{15}$, um die gemeinsamen Werbemaßnahmen abzustimmen, war die Zusammenarbeit keineswegs reibungslos. So entwickelte sich in der Folgezeit eine Kontroverse zwischen den beteiligten Ressorts in Berlin, die längere Zeit andauerte und letztlich den erhofften Erfolg der Aktion erheblich beeinträchtigte. So hatte der Minister für Arbeit vorgeschlagen, sogenannte Landjugendbrigaden zu bilden, die außerhalb der bereits bestehenden Organisationsstrukturen in den Dörfern (also neben den Landwirtschaftlichen Produktionsgenossenschaften und den Volkseigenen Gütern) aufgebaut werden sollten. Dadurch erhoffte sich Minister Macher offensichtlich eine rasche Mobilisierung zusätzlicher Arbeitskräfte, wobei deutliche Kritik an der mangelhaften Kooperation auf seiten der bestehenden LPG geübt wurde: Diese hätten sich nämlich unter Verweis auf „ökonomische Gründe“ bisher geweigert, Jugendliche als Lehrlinge einzustellen, „weil man die Ansicht vertritt, daß die Genossenschaften nicht in der Lage sind, die Jugendlichen zugleich gesellschaftlich und moralisch zu beeinflussen und zu erziehen "16. Der Staatssekretär für Berufsausbildung Rudi Wießner sprach sich gegen diesen Vorschlag aus und betonte, daß es nicht richtig sei, das Arbeitskräfteproblem in der Landwirtschaft auf diese Weise zu lösen. Er versuchte, die Verantwortung dem Landwirtschaftsministerium zuzuschieben: Die Genossenschaften müßten davon überzeugt werden, „Lehrlinge einzustellen, auszubilden und zu jungen Genossenschaftsbauern im Kollektiv der Genossenschaft zu erziehen" 17 .

Zur besseren Koordinierung wurde vermutlich im Sommer 1955 eine Zentrale Kommission gebildet ${ }^{18}$, in der Mitarbeiter nahezu sämtlicher Ministerien vertreten waren. Die Arbeit der Kommission beschränkte sich jedoch im wesentlichen auf die Durchführung von Werbekampagnen, bei der auch die Massenorganisationen (vor allem FDGB und FDJ) beteiligt wurden ${ }^{19}$, sowie auf die Zusammenstellung von Instrukteurgruppen, welche die Aktion zu überwachen hatten ${ }^{20}$. Bis Anfang August 1955 konnten einem Bericht zufolge, den das Sekretariat des ZK in Auftrag gegeben hatte, rund 20700 „Industriekader“ auf dem Lande eingesetzt

13 BAB, DE 1/8739, Bl. 72-74, hier Bl. 73, Bericht der SPK (HA Planung der Arbeitskräftc) vom 30.6. 1955.

1t SAPMO, DY 34, 45/143/6058, Hausmitteilung des FDGB-Bundesvorstandes vom 6. 8. 1955.

$15 \mathrm{Vgl}$. BAB, DQ 2/2124, Vereinbarung zwischen dem Ministerium für Allgemeinen Maschinenbau und dem Ministerium für Land- und Forstwirtschaft.

16 BAB, DQ 2/2123, Wießner am 19. 10. 1955 an Minister Macher.

17 Ebenda.

18 Vgl. BAB, DQ 2/2124, Bericht der Abt. Arbeitskraftlenkung vom 1. 8. 1955.

19 Vgl. BAB, DQ 2/2123, Protokoll über die Sitzung der zentralen Kommission der Aktion „Industriearbeiter aufs Land" am 20. 2. 1956.

20 Vgl. ebenda, Protokoll über die Sitzung der zentralen Kommission der Aktion „Industriearbeiter aufs Land" am 3. 9. 1956. 
werden $^{21}$. Eine Aufschlüsselung nach den Berufsgruppen, die unter anderem Rückschlüsse auf die Qualifizierung zuläßt, zeigt deutlich, daß die Mehrzahl in unteren und mittleren Positionen des landwirtschaftlichen Sektors neu einsteigen mußte. Die mit Abstand größte Gruppe waren die Landarbeiter (9500), gefolgt von 2000 Traktoristen und etwa 1000 Bauarbeitern bzw. Handwerkern. Das Ziel, mit Hilfe der „Industriekader" die Umstrukturierung der Landwirtschaft zu beschleunigen, war dagegen nicht im erhofften Maße erreicht worden ${ }^{22}$. Der Bericht kritisierte daher auch, daß die Aktion „Industriearbeiter aufs Land“ noch zu sehr als „Arbeitskräfteumgruppierung“ angesehen und nicht erkannt werde, „welche große Hilfe ausgewählte politische und fachliche Kader aus der Industrie für die Entwicklung der Landwirtschaft bedeuten".

Mit der Gewinnung von Arbeitskräften für die Landwirtschaft traten wiederum Schwierigkeiten bei der Versorgung mit Wohnraum auf, die von den beteiligten Ministerien im Vorfeld nicht berücksichtigt worden waren. Die Kommunalverwaltung wurde deshalb kurzfristig angewiesen, für die zugewanderten Arbeiter Wohnungen zur Verfügung zu stellen ${ }^{23}$. Als entscheidendes Hindernis erwies sich die Bezahlung, da sich vor allem die Industriearbeiter stets in höheren Lohngruppen als die Landarbeiter befanden. Die berufliche Umgruppierung in die Landwirtschaft war für sie automatisch mit einem Einkommensverlust verbunden. Angesichts der zusätzlichen Anreize wie etwa der Prämien, mit denen Großbaustellen Arbeitskräfte anwerben konnten, hatte die Landwirtschaft in der Zeit zuvor immer mehr an Attraktivität verloren ${ }^{24}$. Das Ministerium für Landund Forstwirtschaft hatte zwar Ende November 1955 die Gewährung eines Lohnausgleiches angekündigt, allerdings dabei eine monatliche Höchstgrenze von $500 \mathrm{DM}$ festgesetzt ${ }^{25}$.

Der Bericht der zentralen Instrukteurgruppe über die Durchführung der Umsetzungsaktion fiel für das Jahr 1956 sehr ernüchternd aus ${ }^{26}$. Der Bedarf an 20000 landwirtschaftlichen Arbeitskräften, der auf die Industrieministerien und Massenorganisationen aufgeteilt worden war, konnte am Jahresende nicht befriedigt werden. So hatte das Ministerium für Schwermaschinenbau von 2412 nur $1850 \mathrm{Ar}$ beitskräfte zur Verfügung stellen können; das Ministerium für Leichtindustrie hatte bei einer Auflage von 2670 sogar nur 987 Arbeiter bereitgestellt. Das Ministerium für Allgemeinen Maschinenbau konnte überhaupt kein Ergebnis vorlegen. Unterschiedlich wurde auch die Mitarbeit der beiden Massenorganisationen bewertet: Während die Instrukteurgruppe die Tätigkeit der FDJ insgesamt recht positiv einschätzte, stufte sie die Mitarbeit des FDGB „bis auf einige Ausnahmen [als] ungenügend" ein. Insgesamt beanstandeten die Instrukteure die mangelnde

21 SAPMO, DY 30/J IV 2/3/483, Bl. 4 und 34-41, hier Bl. 34, Anlage zum Protokoll der Sitzung des Sekretariats des ZK vom 10.8.1955.

22 Der Bericht zählte 46 MTS-Direktoren, 19 Betriebsleiter für VEG, 213 LPG-Vorsitzende, 65 Leiter der ÖLB und 114 Technische Leiter für MTS und VEG auf. Ebenda.

23 W. Stechert, Die Sicherung der Unterbringung der Arbeitskräfte in der Landwirtschaft - eine Hauptaufgabe der Wohnraumlenkung, in: Arbeit und Sozialfürsorge 1955, S. 393 f. und S. 425 f.

24 BAB, DQ 2/2123, Protokoll über die Sitzung der Zentralen Kommission der Aktion „Industricarbeiter aufs Land" am 24. 9. 1956, S. 3.

25 Ebenda, Verfügungen und Mitteilungen des Ministeriums für Land- und Forstwirtschaft vom 28. 11. 1955, S. 2.

26 Zum folgenden: SAPMO, DY 34/22301, Analyse der Instrukteurgruppe vom 21. 12. 1956. 
Vorbereitung des Arbeitseinsatzes der Industriearbeiter und verwiesen dabei besonders auf die nach wie vor bestehende mangelhafte Wohnraumversorgung. Im Sommer 1957 wurde die Zahl der Mitglieder bei der Zentralen Kommission erheblich reduziert und gleichzeitig das Ministerium für Land- und Forstwirtschaft mit der weiteren Federführung beauftragt ${ }^{27}$. Das personell verkleinerte Gremium beschloß kurz darauf, die Aktion „Industriearbeiter aufs Land“ nicht mehr fortzusetzen ${ }^{28}$. Statt dessen sollten nur noch die Landwirtschaftlichen Produktionsgenossenschaften in Mecklenburg bevorzugt mit Arbeitskräften versorgt werden.

\section{Abbau von Arbeitsplätzen im Uranbergban}

Als weiterer Testfall für die DDR-Planwirtschaft erwies sich die berufliche Unterbringung von entlassenen Wismut-Arbeitern ab Spätsommer 1956. Diese Entwicklung hing direkt zusammen mit der zunehmenden Mechanisierung sowie der Stillegung unrentabler Schachtanlagen im Erzgebirge, die seit Mitte der fünfziger Jahre eine deutliche Reduzierung des Belegschaftsstandes verursacht hatten ${ }^{29}$. Informationen der Generaldirektion der SDAG Wismut zufolge sollten bis Ende 1956 sechs Schächte und zwei Aufbereitungsbetriebe stillgelegt werden; das bedeutete die Entlassung von rund 5800 Arbeitskräften, von denen nur ein geringer Teil in anderen Objekten des Uranbergbaus untergebracht werden konnte ${ }^{30}$. Die Arbeitsverwaltung war auf diese Entwicklung nicht vorbereitet; erneut mußte sie sich mit der Bewältigung der sozialen und wirtschaftlichen Folgen auseinandersetzen. Auf Veranlassung des Sekretariats des ZK der SED sollten die Staatliche Plankommission, die Industrieministerien sowie das Arbeitsministerium Vorschläge ausarbeiten, „welche industriellen Objekte im Wismutgebiet aufgebaut werden können, um die bestehenden Anlagen auszunutzen und die Arbeitskräfte, die dort seßhaft sind [...] zu beschäftigen" ${ }^{31}$. Die Kaderleiter der SDAG Wismut planten zunächst, die zur Entlassung anstehenden Arbeiter innerhalb des Unternehmens zu versetzen ${ }^{32}$. Das Berliner Arbeitsministerium setzte schließlich doch eine andere Strategie durch, da die Beschäftigtenzahl im Uranbergbau langfristig gesenkt werden mußte und somit eine Umgruppierung innerhalb der SDAG Wismut nicht in Frage kam. Statt dessen sollten die Arbeitskräfte an große volkseigene Betriebe der Schwer- und Grundstoffindustrie in der gesamten DDR vermittelt werden ${ }^{33}$. Die Bereitstellung von neuen Arbeitsplätzen blieb anfangs jedoch

27 Ebenda, Ministerium für Land- und Forstwirtschaft an den FDGB-Bundesvorstand (Juli 1957).

28 Ebenda, Kurze Information über die Beratung zur Sicherung des Arbeitskräftebedarfs für die Landwirtschaft im Ministerium für Land- und Forstwirtschaft am 16. 7. 1957.

29 Karlsch, Der Aufbau der Uranindustrien in der SBZ/DDR und ČSR als Folge der sowjetischen "Uranlücke“, S. 15.

30 SAPMO, DY 30/J IV 2/3 A/532, Informationen der ZK-Abt. Grundstoffindustrie vom 25. 8. 1956. Vertreter der Generaldirektion der SDAG Wismut hatten diesen Sachverhalt auf einer Besprechung am 24. 8. 1956 mitgeteilt, an der der 1. Sekretär der SED-Gebietsparteileitung Wismut (Alois Bräutigam) und jeweils ein Vertreter des Zentralvorstandes der IG Wismut, des Ministeriums für Arbeit und der ZK-Abt. Grundstoffindustrie (Bertold Handwerker) teilnahmen.

31 SAPMO, DY 30/J IV 2/3/527, Bl. 2, Protokoll der Sitzung des Sekretariats vom 5. 9. 1956.

32 BAB, DQ 2/2104, Aktenvermerk des VEB Bau-Unicn Magdeburg vom 23. 10. 1956.

33 Ebenda. 
eine Aufgabe der SDAG Wismut ${ }^{34}$ sowie der betroffenen Bezirks- und Kreisverwaltungen, während sich andere Großbetriebe und Bezirksverwaltungen auffallend zurückhielten.

Die Bezirksverwaltungen im Erzgebirge waren mit der arbeitsmarktpolitischen Aufgabe bald überfordert und baten die Staatliche Plankommission Anfang 1957 um Unterstützung ${ }^{35}$. Das Ministerium für Arbeit und Berufsausbildung beschränkte seine Aktivitäten zunächst darauf, Mitarbeiter in die betroffenen Kreise zu entsenden, um die Zahl der Entlassungen sowie die beruflichen „Umsetzungen " $^{\text {" zu registrieren }}{ }^{36}$. Eine Lenkung und Steuerung war zu diesem Zeitpunkt nicht erkennbar. Dies änderte sich erst Anfang März, nachdem das Sekretariat des ZK der SED beschlossen hatte, eine Kommission unter Vorsitz von Minister Macher zu bilden, die „geeignete Vorschläge zur anderweitigen Beschäftigung dieser freiwerdenden Arbeitskräfte" ausarbeiten sollte ${ }^{37}$. Gleichzeitig legte das SEDFührungsgremium fest, daß die entlassenen Arbeiter entweder in andere Betriebe innerhalb und außerhalb des Bezirkes Karl-Marx-Stadt zu vermitteln seien oder durch Verlegung von Betriebsteilen bzw. Errichtung zusätzlicher Produktionsstätten neue Arbeitsplätze finden sollten.

Trotz dieser Beschlüsse ging die berufliche Wiedereingliederung der entlassenen Arbeiter des Uranbergbaus nur langsam voran. Neue Beschäftigungsmöglichkeiten in nennenswertem Umfange boten nach Ansicht der Staatlichen Plankommission der Steinkohlenbergbau ${ }^{38}$ sowie das neue industrielle Großprojekt ,Schwarze Pumpe', die jedoch nicht alle arbeitslosen Wismut-Arbeiter aufnehmen konnten. Die einseitige Ausrichtung auf den Uranbergbau schloß eine neue berufliche Perspektive für die Mehrzahl der entlassenen Arbeiter in den Bezirken des Erzgebirges weitgehend aus ${ }^{39}$. Eine reibungslose Umgruppierung wurde somit unwahrscheinlich; mit einem Ansteigen der Arbeitslosenzahlen mußte gerechnet werden. Das Ministerium für Arbeit und Berufsausbildung ging für das Jahr 1957 von insgesamt 17000 Entlassungen aus; besonders hart betroffen waren die Stadtkreise Johanngeorgenstadt (4200), Schneeberg (6800) sowie der Kreis Auerbach $(2700)^{40}$. Darüber hinaus zeigte sich schon frühzeitig, daß zahlreiche Bergarbeiter eine neue Beschäftigung mit geringeren Verdienstmöglichkeiten wie etwa in der Textil- und Papierindustrie oder der Landwirtschaft ablehnten: „Durch die bei der SDAG Wismut eingeführten zahlreichen Vergünstigungen [...] sind die Kumpels

34 BAB, DQ 2/2104, Abt. Arbeitskraftlenkung am 4.1. 1957 an den Rat des Bezirkes Magdeburg (Abt. Arbeit und Berufsausbildung).

35 SAPMO, NY 4090/359, Bl. 88, Rat des Bezirkes Karl-Marx-Stadt am 7. 2. 1957 an den Vorsitzenden der Staatlichen Plankommission Bruno Leuschner.

36 BAB, DQ 2/2129, Vermerk der Abt. Arbeitskraftlenkung vom 18. 2. 1957.

37 BAB, DQ 2/1698, Stellvertretender Ministerpräsident Fritz Selbmann am 8.3. 1957 an Minister Macher. Der Kommission sollten "verantwortliche" Vertreter der Staatlichen Plankommission, des Ministeriums für Allgemeinen Maschinenbau, des Ministeriums für Leichtindustrie, des FDGB-Bundesvorstandes, des Rates des Bezirkes Karl-Marx-Stadt und der Generaldirektion der SDAG Wismut angehören.

38 BAB, DE 1/413, Bl. 6-11, hier Bl. 6, Bericht der Staatlichen Plankommission über die Arbeitskräftelage im Wismut-Gebiet Johanngeorgenstadt vom 22. 9. 1956.

$39 \mathrm{BAB}, \mathrm{DC} 1 / 1063$, Bericht (Entwurf) der ZKSK über die Lage und die notwendigen Veränderungen der Wirtschaftsstruktur im Bergbaugebiet der SDAG Wismut [Mitte März 1957], S. 3.

40 $\mathrm{BAB}, \mathrm{DQ} 2 / 2129$, Bericht der Abt. Arbeitskraftlenkung vom 15. 3. 1957, S. 1. 
gegenüber den Beschäftigten in anderen Industriezweigen verwöhnt worden. “" Als weiteres Hindernis erwies sich erneut die mangelhafte Wohnraumversorgung, die der Arbeitskräftelenkung auch in diesem Fall im Wege stand. Auf Anweisung des Sekretariats des ZK der SED stellte Arbeitsminister Macher schließlich eine Kommission zusammen, die geeignete Beschäftigungspläne ausarbeiten sollte ${ }^{42}$. Der Handlungsspielraum war jedoch eng gesetzt, wie der Leiter der Hauptabteilung Investitionen bei der Staatlichen Plankommission, Friedrich Lange ${ }^{43}$, bereits auf der ersten Sitzung hervorhob. So durften sich die Neuinvestitionen im Zusammenhang mit der beruflichen Eingliederung von entlassenen Wismut-Arbeitern nur im Rahmen des bereits laufenden Volkswirtschaftsplanes 1957 bewegen. Über den Plan hinausgehende Investitionen konnten deshalb nicht mehr vorgenommen werden. Alle Ministerien sollten daher überprüfen, „welche bereits vorhandenen Produktionskapazitäten in die Wismut-Kreise verlagert werden können “44. Darüber hinaus befürwortete die Mehrheit der Kommissionsmitglieder eine Weiterleitung der „freigewordenen Arbeitskräfte“ in die Landwirtschaft. Mit der kurz zuvor eingeleiteten Aktion „Industriearbeiter aufs Land“ schien sich eine Lösung anzubahnen. Diesem Vorschlag widersprach nur das Ministerium für Land- und Forstwirtschaft, auf das nunmehr größere Koordinierungsaufgaben zukamen ${ }^{45}$. Für die Landwirtschaft konnten aufgrund des bereits beschriebenen Scheiterns dieser Aktion nur äußerst wenige Bergarbeiter gewonnen werden: 1956610 und im I. Quartal 1957250 Arbeitskräftet6.

Die Tatsache, daß es sich bei der SDAG Wismut um ein Unternehmen handelte, das nach wie vor unter maßgeblichem sowjetischen Einfluß stand, erschwerte zusätzlich die langfristige Planung. So wurde die deutsche Arbeitsverwaltung spät und unzureichend über die Zahl der entlassenen Wismut-Arbeiter und deren berufliches Qualifikationsprofil informiert. Der ZKSK-Vorsitzende empfahl daraufhin dem Arbeitsminister, „eine enge Verbindung mit der Generaldirektion der SDAG Wismut zu halten" ${ }^{47}$. Neben dem Ministerium für Land- und Forstwirtschaft setzten auch die übrigen an der Aktion beteiligten Ministerien eigene „Werber" im Erzgebirge ein, um Arbeitskräfte zu vermitteln. Sicherheitsbedenken von seiten der sowjetischen Werksleitungen verhinderten allerdings oftmals schon den

+1 BAB, DC 1/1063, Bericht (Entwurf) der ZKSK über die Lage und die notwendigen Veränderungen der Wirtschaftsstruktur im Bergbaugebiet der SDAG Wismut [Mitte März 1957], S. 3.

42 SAPMO, DY 34, 45/143/6058, Minister Macher am 16. 3. 1957 an den Sekretär für Lohnfragen beim FDGB-Bundesvorstand, Otto Lehmann. Die Kommission setzte sich zusammen aus Vertretern der Staatlichen Plankommission, der Staatlichen Geologischen Kommission, des Ministeriums für Kohle und Energie, des Ministeriums für Allgemeinen Maschinenbau, des Ministeriums für Leichtindustrie, des Ministeriums für Berg- und Hüttenwesen, des Ministeriums für Landund Forstwirtschaft, des Ministeriums für Aufbau, des Staatssekretariats für Örtliche Wirtschaft, des FDGB-Bundesvorstandes, der SDAG Wismut, des Rates des Bezirkes Karl-Marx-Stadt, des Rates der Stadt Johanngeorgenstadt und des Amtes für Technik. BAB, DQ 2/1251, Protokoll über dic 1. Sitzung der Kommission für die Umsetzung der freiwerdenden Arbeitskräfte bei der SDAG Wismut am 22. 3. 1957, S. 1.

t3 Angaben zu Lange in: SBZ-Handbuch, S. 962.

$44 \mathrm{BAB}, \mathrm{DQ} 2 / 1251$, Protokoll über die 1 . Sitzung der Kommission für die Umsetzung der freiwerdenden Arbeitskräfte bei der SDAG Wismut am 22. 3. 1957, S. 2.

t5 Ebenda, S. 3.

t6 $\mathrm{BAB}, \mathrm{DQ} 2 / 2129$, Protokoll über die 2. Sitzung der Kommission für die Umsetzung der freiwerdenden Arbeitskräfte bei der SDAG Wismut am 5. 4. 1957.

t7 BAB, DQ 2/1698, ZKSK-Vorsitzender am 3.5. 1957 an Minister Macher, S. 2. 
Zugang zu den Schachtanlagen ${ }^{48}$. Nach Angaben von Arbeitsminister Macher konnte ein großer Teil der Bergarbeiter, deren Entlassung für 1957 geplant war, bereits im Juni ein neues Beschäftigungsverhältnis vorweisen. Damit hatte sich die Situation leicht entspannt, und Macher hielt sogar das Weiterbestehen der Kommission "nicht mehr für erforderlich" 49 .

Der Stein- und Braunkohlenbergbau befand sich Mitte der fünfziger Jahre in einer strukturellen Krise. Die ohnehin sehr begrenzten Steinkohlevorkommen gingen sichtbar zur Neige, so daß in diesem Bereich mit einem Anstieg der Erwerbstätigen nicht zu rechnen war. Darüber hinaus hatte das Staatssekretariat für Staatssicherheit am 18. November 1954 auf erhebliche Fehlmengen bei der Kohleförderung hingewiesen ${ }^{50}$. Als wesentliche Ursachen wurden Havarien und die nicht rechtzeitige Durchführung von Bauvorhaben genannt. Das Staatssekretariat, das in der zweiten Hälfte der fünfziger Jahre immer stärker die Gesamtwirtschaft zu kontrollieren versuchte, sich dabei aber zunächst auf Kaderfragen und Spionageangelegenheiten beschränkte ${ }^{51}$, machte für die "häufig aufgetretenen Zugunfälle [...] feindliche Elemente im Auftrage der imperialistischen Geheimdienste" verantwortlich52. Die SED-Führung reagierte im Herbst 1956, befürchtete sie doch negative Folgen für die industrielle Produktion und mittelbar auch für den Arbeitsmarkt. Das Politbüro ging nämlich davon aus, daß sich die Brennstoff-Reserve von 29 Tagen (1955) auf acht Tage reduzieren würde53. Zur Sicherstellung der Versorgung mit Kohle nahm das Politbüro Verhandlungen mit den Mitgliedstaaten des Rates für gegenseitige Wirtschaftshilfe (RGW) auf, um die Kohlezulieferungen zu erhöhen ${ }^{54}$, die vor allem aus Polen stark ins Stocken geraten waren. Dadurch war letztlich auch die Produktion in den Stahl- und Walzwerken äußerst gefährdet. Außerdem wurden die Bergarbeiter in der DDR zur Steigerung ihrer Arbeitsleistung mobilisiert. Das Sekretariat des ZK der SED beschloß in dem Zusammenhang am 29. März 1957, eine „zentrale Kohle- und Energiekonferenz" durchzuführen, an der Wirtschaftsfunktionäre, Mitglieder der Parteileitungen und der Gewerkschaften teilnehmen sollten ${ }^{55}$. Kurz zuvor hatte der Ministerrat der DDR am 21. März 1957 das Kohle- und Energieprogramm beschlossen ${ }^{56}$. In dem Zusammenhang entwickelte die ZK-Abteilung Industrie einen „Plan zur Mobilisierung aller Werktätigen zur Erfüllung des Kohle- und Energieprogramms unserer Republik" ${ }^{77}$, den das Sekretariat des ZK der SED grundsätzlich bestätigte ${ }^{58}$.

48 Ebenda, Vermerk der Abt. Arbeitskraftlenkung vom 6. 5. 1957.

49 Ebenda, Macher an den stellvertretenden Vorsitzenden des Ministerrates Fritz Selbmann, Juni 1957.

50 BStU, MfS, DA 55/54, S. 2.

51 Vgl. Anatomie der Staatssicherheit. Die Hauptabteilung XVIII.

52 BStU, MfS, DA 55/54, S. 2.

53 SAPMO, DY 30/J IV 2/2/508, Bl. 6 und 8-30, hier Bl. 8, Protokoll der Sitzung des Politbüros am 30. 10. 1956.

54 SAPMO, DY 30/J IV 2/3/551, Bl. 1, Protokoll der Sitzung des Sekretariats des ZK am 6. 3. 1957.

55 SAPMO, DY 30/J IV 2/3/554, Bl. $7 \mathrm{f}$.

56 Hübner, Zum Kohle- und Energieprogramm der DDR 1957.

57 SAPMO, DY 30/J IV 2/3 A/562, Vorlage vom 8. 4. 1957.

58 SAPMO, DY 30/J IV 2/3/557, Protokoll der Sitzung des Sekretariats des ZK vom 17.4. 1957; SAPMO, DY 30/J IV 2/3/563, Bl. 9, Protokoll der Sitzung des Sekretariats des ZK vom 29. 5. 1957. 
Die Kohlegewinnung sollte also insgesamt enorm gesteigert werden. Hintergrund dafür war unter anderem auch der Aufbau des Braunkohleveredelungswerkes ,Schwarze Pumpe' bei Hoyerswerda. Eine Folge des Kohle- und Energieprogramms war der verstärkte Einsatz von Investitionsmitteln und Arbeitskräften in diesem Wirtschaftszweig, wodurch sich unter anderem das geplante Arbeitskräftekontingent vergrößerte. Das Kohle- und Energieprogramm kann als Versuch angesehen werden, einzelne zentrale Bereiche der Schwerindustrie wieder bevorzugt mit Investitionsmitteln und Arbeitskräften zu versorgen. Ende der fünfziger Jahre begann die Chemische Industrie an Bedeutung zu gewinnen. Dabei setzte ein Anstieg der Bruttoproduktion ein, der bald über dem der übrigen Bereiche der Grundstoffindustrie lag59. Die "Chemisierung" der Wirtschaft ${ }^{60}$ koppelte die SED-Führung in Absprache mit dem RGW Ende der fünfziger bzw. Anfang der sechziger Jahre mit verstärkten Bemühungen um eine eigene Erdöl- und Erdgasgewinnung, die jedoch angesichts der geringfügigen Vorräte nahezu erfolglos blieb.

Tabelle 32: Entwicklung des Arbeitskräftebestandes in den Betrieben der HV Stein- und Braunkoble (1955/56)

\begin{tabular}{lllllll}
\hline Jahr & $\begin{array}{l}\text { Gesamt- } \\
\text { beschäftigte } \\
\text { der HV } \\
\text { Steinkohle }\end{array}$ & $\begin{array}{l}\text { Zu- } \\
\text { gänge }\end{array}$ & $\begin{array}{l}\text { Ab- } \\
\text { gänge }\end{array}$ & $\begin{array}{l}\text { Gesamt- } \\
\text { beschäftigte } \\
\text { der HV } \\
\text { Braunkohle }\end{array}$ & $\begin{array}{l}\text { Zu- } \\
\text { gänge }\end{array}$ & $\begin{array}{l}\text { Ab- } \\
\text { gänge }\end{array}$ \\
\hline 1955 & 26230 & 5714 & 7320 & 111135 & 19288 & 20840 \\
1956 & 25927 & 5965 & 6269 & 109499 & 17696 & 17186 \\
\hline
\end{tabular}

Quelle: BAB, DQ 2/1698, Bericht der Abt. Arbeitskraftlenkung vom 15. 4. 1957.

Der Rückgang der Beschäftigtenzahlen im Stein- und Braunkohlenbergbau hatte bis 1956 trotz vielfältiger Bemühungen der beteiligten Fachministerien letztlich nicht aufgehalten werden können. Mit der beschäftigungspolitischen Offensive, die primär auf das Ziel zurückzuführen war, die Fördermengen an Braun- und Steinkohle zu erhöhen, vergrößerte sich auch der Belegschaftsstand. Bei den Betrieben der Hauptverwaltung Braunkohle wurden im 1. Halbjahr 1957 bereits 117369 Beschäftigte gezählt ${ }^{6}{ }^{1}$. Bei der Hauptverwaltung Steinkohle war dagegen die Zahl der Beschäftigten nicht gestiegen, sondern weiter auf 25193 zurückgegangen ${ }^{62}$. Damit blieben die Möglichkeiten begrenzt, alle entlassenen Wismut-Arbeiter in diesen Bergbauzweigen beruflich zu integrieren.

Die Stillegung von Schachtanlagen der SDAG Wismut und die damit zusammenhängende Verkleinerung der Belegschaft war im Sommer 1957 noch keines-

\footnotetext{
59 Roesler, Die Herausbildung der sozialistischen Planwirtschaft in der DDR, S. 288.

$60 \mathrm{Vgl}$. Karlsch, Der Traum vom Öl, S. 73.

61 BAB, DQ 2/1698, Analyse vom 19. 8. 1957, S. 2.

62 Ebenda.
} 
wegs abgeschlossen, sondern setzte sich auch im Herbst weiter fort ${ }^{63}$. In einigen Schachtanlagen ergab sich nach Mitteilung der Wismut-Generaldirektion ein neuer Arbeitskräfteüberhang von insgesamt 4650 Personen. Die beteiligten Ministerien sowie die Regional- und Lokalverwaltungen sahen sich somit erneut mit der Aufgabe konfrontiert, Beschäftigungsmöglichkeiten für die entlassenen Arbeiter anzubieten. Größere Betriebe der volkseigenen Industrie, in erster Linie wieder der Stein- und Braunkohlebergbau sowie die Bauindustrie, sollten zusätzliche Arbeitsplätze anbieten. Darüber hinaus planten die beteiligten Stellen eine Umsetzung der nicht ortsansässigen Arbeitskräfte. Wismut-Arbeiter, deren Wohnsitz außerhalb des Erzgebirges lag, sollten in ihre Heimatgebiete zurückkehren. Damit wurde das Problem der beruflichen Unterbringung aus Sicht der SDAG Wismut externalisiert, da auf diese Weise sämtliche DDR-Bezirke zur Bewältigung des Arbeitsmarktproblems herangezogen wurden. Die langfristige Planung wurde aber auch in diesem Fall dadurch behindert, daß die Kommunalverwaltungen in den betroffenen Kreisen der SDAG Wismut im Januar 1958 über keine konkreten Zahlenangaben verfügten ${ }^{64}$. Von einer neuen Entlassungswelle waren nach Angaben der Hauptabteilung Kader (SDAG Wismut) bis zum Ende des I. Quartals 1958 rund 5500 Arbeiter betroffen ${ }^{65}$. Besonders schwierig gestaltete sich die Lage in Johanngeorgenstadt, da es sich bei den zur Entlassung anstehenden Arbeitskräften (2000) nur um Ortsansässige handelte. Da die Aufnahmekapazitäten für zusätzliche Arbeitskräfte in den dortigen volkseigenen Betrieben "sehr gering" waren, stieg die Zahl der Arbeitsuchenden, "wodurch eine noch nicht dagewesene Unzufriedenheit [in] der Bevölkerung entstanden“ sei66.

Die Kommunalverwaltungen wurden von der Generaldirektion der SDAG Wismut - darauf ist bereits hingewiesen worden - spät über die konkrete Zahl der Entlassungen informiert. Ebenso problematisch erwies sich die statistische Erfassung über die berufliche Umsetzung dieser Wismut-Arbeiter, was ursächlich mit den stark eingeschränkten Möglichkeiten der Arbeitsämter zusammenhing. Diese waren 1951 als selbständige Dienststellen aufgelöst worden und hatten eine ganze Reihe von zentralen Aufgaben an die Kreisverwaltungen bzw. ab Sommer 1952 an die Bezirksverwaltungen abtreten müssen ${ }^{67}$. Darüber hinaus war die Arbeitskräftewerbung grundsätzlich den Betrieben übertragen worden. Damit hatte letztlich die Arbeitsverwaltung ihre koordinierende Funktion verloren. Das erklärt auch die Notiz einer ZKSK-Mitarbeiterin Mitte Februar 1958: „Wo die restlichen ca. 1500 Arbeitskräfte verblieben sind, ist nicht bekannt, da es keine Möglichkeit gibt, die Arbeitskräfte zu registrieren.“"68

$63 \mathrm{BAB}, \mathrm{DQ} 2 / 1698$, Bericht der Abt. Arbeitskraftlenkung vom 11.11. 1957 über die Lage bei der SDAG Wismut, S. 1.

64 BAB, DQ 2/2129, Niederschrift über die am 23.1. 1958 stattgefundene Besprechung mit kommunalen Abteilungsleitern (Abt. für Arbeit).

65 Ebenda, Bericht der Abt. Arbeitskraftlenkung vom 27. 1. 1958.

66 Ebenda, Stellvertreter des Generaldirektors der SDAG Wismut, R. Schröder, am 31.1.1958 an Minister Macher, S. 2.

67 Verordnung über die Aufgaben der Arbeitsverwaltungen und über die Lenkung der Arbeitskräfte vom 12. 7. 1951, in: Gesetzblatt der DDR 1951, S. 687-689. Frerich/Frey bezeichnen die Arbeitsämter fälschlicherweise als „Sonderbehörden“. Frerich/Frey, Sozialpolitik in der DDR, S. 176.

68 BAB, DC 1/1063, Notiz der Kontrolleurin Ruth K. (ZKSK, Arbeitsgruppe Arbeit und Berufsausbildung) vom 18. 2. 1958. 